\title{
EXPERIMENTAL STUDY OF ELECTRONIC QUANTUM INTERFERENCE, PHOTONIC CRYSTAL CAVITY, PHOTONIC BAND EDGE EFFECTS FOR OPTICAL AMPLIFICATION
}

Shawn-Yu Lin

Rensselaer Polytechnic Institute 110 8th Street

Troy, New York 12180

26 Jan 2016

Final Report

APPROVED FOR PUBLIC RELEASE; DISTRIBUTION IS UNLIMITED.

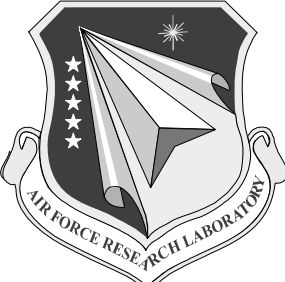

AIR FORCE RESEARCH LABORATORY Space Vehicles Directorate 3550 Aberdeen Ave SE AIR FORCE MATERIEL COMMAND KIRTLAND AIR FORCE BASE, NM 87117-5776 


\section{DTIC COPY \\ NOTICE AND SIGNATURE PAGE}

Using Government drawings, specifications, or other data included in this document for any purpose other than Government procurement does not in any way obligate the U.S. Government. The fact that the Government formulated or supplied the drawings, specifications, or other data does not license the holder or any other person or corporation; or convey any rights or permission to manufacture, use, or sell any patented invention that may relate to them.

This report is the result of contracted fundamental research deemed exempt from public affairs security and policy review in accordance with SAF/AQR memorandum dated 10 Dec 08 and AFRL/CA policy clarification memorandum dated 16 Jan 09. This report is available to the general public, including foreign nationals. Copies may be obtained from the Defense Technical Information Center (DTIC) (http://www.dtic.mil).

AFRL-RV-PS-TR-2016-0003 HAS BEEN REVIEWED AND IS APPROVED FOR
PUBLICATION IN ACCORDANCE WITH ASSIGNED DISTRIBUTION STATEMENT.

//SIGNED//

DAVID CARDIMONA

Program Manager
//SIGNED//

PAUL D. LEVAN, Ph.D.

Technical Advisor, Space Based Advanced Sensing and Protection

//SIGNED//

JOHN BEAUCHEMIN

Chief Engineer, Spacecraft Technology Division

Space Vehicles Directorate

This report is published in the interest of scientific and technical information exchange, and its publication does not constitute the Government's approval or disapproval of its ideas or findings.

Approved for public release; distribution is unlimited. 


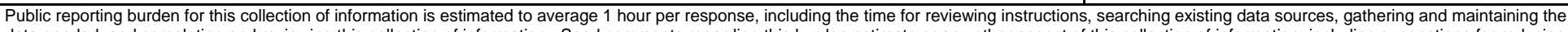

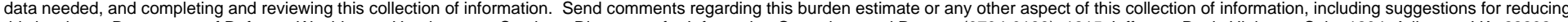

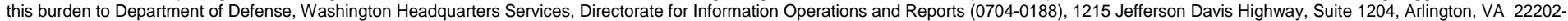

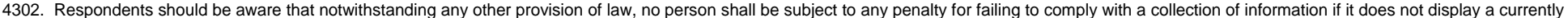
valid OMB control number. PLEASE DO NOT RETURN YOUR FORM TO THE ABOVE ADDRESS.

\begin{tabular}{l|l|l} 
1. REPORT DATE (DD-MM-YY) & 2. REPORT TYPE & 3. DATES COVERED (FrOm - To)
\end{tabular}

26-01-2016 Final Report

\section{TITLE AND SUBTITLE}

Experimental Study of Electronic Quantum Interference, Photonic Crystal Cavity,

Photonic Band Edge Effects for Optical Amplification

7 Nov $2014-11$ Jan 2016

5a. CONTRACT NUMBER

FA9453-15-1-0310

5b. GRANT NUMBER

5c. PROGRAM ELEMENT NUMBER

$62601 \mathrm{~F}$

6. AUTHOR(S)

5d. PROJECT NUMBER

4846

Shawn-Yu Lin

5e. TASK NUMBER

PPM00015091

5f. WORK UNIT NUMBER

EF126057

\section{PERFORMING ORGANIZATION NAME(S) AND ADDRESS(ES)}

8. PERFORMING ORGANIZATION REPORT NUMBER

Rensselaer Polytechnic Institute

110 8th Street

Troy, New York 12180

\section{SPONSORING I MONITORING AGENCY NAME(S) AND ADDRESS(ES)}

Air Force Research Laboratory

Space Vehicles Directorate

3550 Aberdeen Ave., SE

Kirtland AFB, NM 87117-5776

\section{DISTRIBUTION I AVAILABILITY STATEMENT}

Approved for public release; distribution is unlimited.

\section{SUPPLEMENTARY NOTES}

\section{ABSTRACT}

Photonic crystals can produce strong light trapping and enhancement of the field using the tailoring of dispersion and the photonic band gap. The band gap frequency can be matched to tailor the emission from active medium such as quantum dots and quantum wells. In addition, the selective matching of the bandgap can be done to one or two of electronic levels in a multi-level system to influence the emission.

\section{SUBJECT TERMS}

quantum interference, photonic crystals

\begin{tabular}{|c|c|c|c|c|c|}
\hline \multicolumn{3}{|c|}{ 16. SECURITY CLASSIFICATION OF: } & \multirow{2}{*}{$\begin{array}{l}\text { 17. LIMITATION } \\
\text { OF ABSTRACT } \\
\text { Unlimited }\end{array}$} & \multirow{2}{*}{$\begin{array}{l}\text { 18. NUMBER } \\
\text { OF PAGES } \\
20\end{array}$} & \multirow{2}{*}{$\begin{array}{l}\text { 19a. NAME OF RESPONSIBLE PERSON } \\
\text { David Cardimona } \\
\text { 19b. TELEPHONE NUMBER (include area } \\
\text { code) }\end{array}$} \\
\hline $\begin{array}{l}\text { a. REPORT } \\
\text { Unclassified }\end{array}$ & $\begin{array}{l}\text { b. ABSTRACT } \\
\text { Unclassified }\end{array}$ & $\begin{array}{l}\text { c. THIS PAGE } \\
\text { Unclassified }\end{array}$ & & & \\
\hline
\end{tabular}


(This page intentionally left blank)

Approved for public release; distribution is unlimited. 


\section{Table of Contents}

1.0 Summary

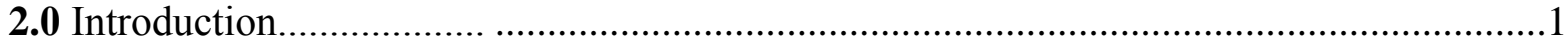

3.0 Methods, Assumptions, and Procedures...................................................................

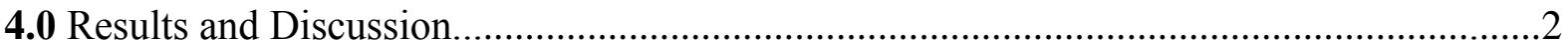

4.1 Design of 3D PBG and Growth of Epitaxial Structure …............................................2

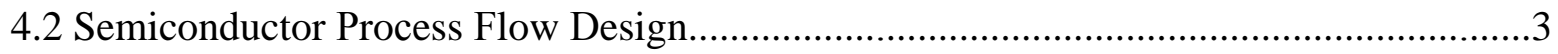

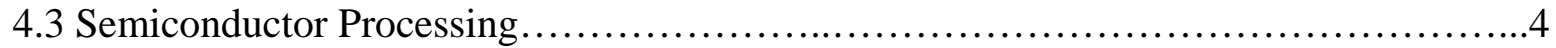

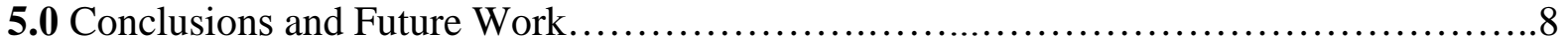

References.................................................................9

Acronyms..................................................................... 


\section{List of Figures}

1. Schematic of the epitaxially grown structure for $3 \mathrm{D}$ photonic crystal. The structure consists of alternating layers of GaAs/AlGaAs which will form the lattice components of the photonic crystal in the growth direction.

2. Process flow for the GaAs-AlGaAs photonic crystal: a) Denotes the dry etching process used to etch GaAs-AlGaAs epitaxially grown material, b) Selective oxidation of AlGaAs layers to AlOx, c) Selective etch of AlOx layers..............................................................

3. Scanning electron microscopy (SEM) image of the fabricated 2D structure using electron beam lithography. ZEP was used as the electron beam resist......................4

4. SEM image of structure after $\mathrm{SiO}_{2}$ etch process calibration. The residual photoresist, $\mathrm{SiO}_{2}$, and underlying $\mathrm{GaAs}$ layer can be seen. A CHF3/O $\mathrm{O}_{2}$ based etch process was

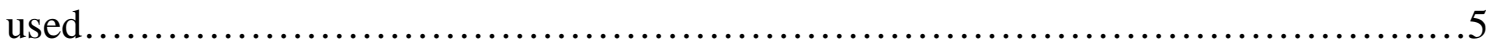

5. Image of the sample after $\mathrm{GaAs}$ etch calibration. An intact hard mask $\left(\mathrm{SiO}^{2}\right)$ and the

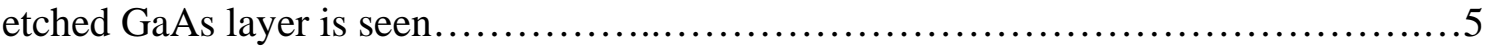

6. Etch rate variation of GaAs calibration samples and the epitaxially-grown GaAs-AlGaAs samples under dry etch process.................................................

7. Oxidation rate of AlGaAs as function of composition. The temperature is kept constant at

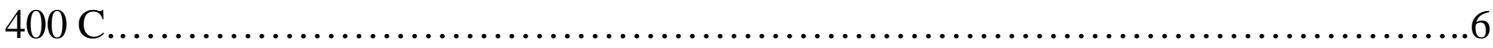

8. Steam oxidation of AlGaAs into GaAs. The samples are kept inside a quartz boat, and N2

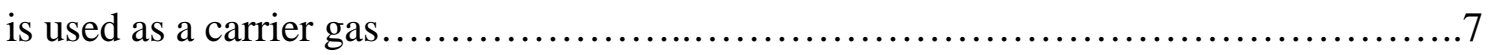

9. SEM image of a lattice of the initial GaAs-AlGaAs photonic crystal sample fabricated. 


\section{ACKNOWLEDGMENTS}

This material is based on research sponsored by Air Force Research Laboratory under agreement number FA9453-15-1-0310. The U.S. Government is authorized to reproduce and distribute reprints for Governmental purposes notwithstanding any copyright notation thereon.

\section{DISCLAIMER}

The views and conclusions contained herein are those of the authors and should not be interpreted as necessarily representing the official policies or endorsements, either expressed or implied, of Air Force Research Laboratory or the U.S. Government. 
(This page intentionally left blank)

iv

Approved for public release; distribution is unlimited. 


\subsection{SUMMARY}

Photonic crystals can produce strong light trapping and enhancement of the field using the tailoring of dispersion and the photonic band gap. The band gap frequency can be matched to tailor the emission from active medium such as quantum dots and quantum wells. In addition, the selective matching of the bandgap can be done to one or two of electronic levels in a multi-level system to influence the emission. Here the goal is to demonstrate a three dimensional photonic bandgap structure amenable to active material inclusion in it with minimal fabrication complexity. Further on the study modification of the absorption profile as a result of the photonic band gap and its experimental validation would be performed.

\subsection{INTRODUCTION}

Three dimensional (3D) photonic crystals and their optical properties have attracted a lot of attention in the past decade. The ability of these structures to confine light in all three dimensions provides for improved light-matter coupling and analysis of the resulting physical phenomena. The band gap frequency of this system can be varied to tailor to the electronic transition levels of a gain medium such as InAs quantum dot or an InGaAs quantum well. The band gap can be varied in addition to include either one or two electronic levels of a multi-level system and to influence the electronic quantum interference. Finally a micro-cavity can be introduced into the photonic crystal for intense light matter interaction. Thus, the ability of these structures to confine and concentrate light provides new opportunities for improved sensitivity, integration and enhanced functionality of devices.

Currently, many of the 3D photonic crystal structures are manufactured by layer to layer lithography or by structure inversion. These techniques are highly fabrication intensive and significant in terms of cost. Moreover, scaling of the lattice and inclusion of active materials is not straightforward. In this project, a 3D photonic crystal with band gap in the near infrared is proposed with minimal fabrication complexity. In addition, use of III-V materials makes this structure highly amenable to embedding of active materials for studying light matter confinement. Use of an epitaxially-grown structure for the third dimension makes the scaling of lattices easier than conventional layer by layer assembly.

\subsection{METHODS, ASSUMPTIONS, AND PROCEDURES}

In the past 12 months, this project has been successful in the design and fabrication of a set of 3D photonic crystal samples using GaAs/AlGaAs materials system for a bandgap of $1 \mu \mathrm{m}$. A growth structure was designed and grown by molecular beam epitaxy. A process flow for the fabrication of the photonic crystal was developed and initial results from this process are very encouraging.

Some of the major milestones of this project are

- Design of a GaAs-AlGaAs based material structure: Using the lattice-matched $\mathrm{GaAs} / \mathrm{AlGaAs}$ structure, a growth structure was designed for the fabrication of multilayer 
3D photonic crystal. This technique does not depend on the layer by layer stacking typically employed for 3D photonic crystals. Since the GaAs-AlGaAs material systems is nearly lattice matched, increasing the number of photonic lattices is now a function of the epitaxial growth as opposed to the fabrication process. A wafer consisting of 4 lattice constants in the vertical direction was grown by molecular beam epitaxy (MBE) for the fabrication of photonic crystal

- Process flow for 3D photonic crystal fabrication: A semiconductor fabrication flow consisting of minimal lithography and etching steps was developed. With a combination of electron beam lithography, dry etching and oxidation steps, the photonic crystal design was explored.

- Development of Etch and Oxidation Techniques: For fabricating the 3D photonic crystal using the GaAs-AlGaAs design, it is necessary to deep etch the GaAs-AlGaAs structure with feature sizes as small as $225 \mathrm{~nm}$. A dual etch step which involved pattern transfer from the resist to oxide, and from oxide to GaAs-AlGaAs was developed for this system. Moreover, the etches developed are highly selective, and are therefore robust and stable with respect to process variations. A technique for oxidation of AlGaAs was also developed without affecting the optical quality the structure.

- Fabrication of photonic crystal structures: Using the techniques mentioned above, two samples of $3 \mathrm{~mm} \times 3 \mathrm{~mm}$ photonic crystal samples were fabricated. The optical testing is currently underway.

\subsection{RESULTS AND DISCUSSION}

\subsection{Design of 3D PBG and Growth of Epitaxial Structure}

In order to have an optically active 3D- photonic crystal, the use of III-V material system with favorable emission properties is desired. An epitaxial design based on GaAs/AlGaAs was designed and epitaxially grown using molecular beam epitaxy. This design is amenable to fabrication of 3D photonic crystal and embedding of active optical material with minimal design variations and complexity. Based on theoretical calculations [1-2], the designed system would be having a bandgap near $1 \mu \mathrm{m}$ wavelength upon fabrication. This wavelength is ideal for strained InGaAs quantum well structures which can be embedded into the GaAs/AlGaAs matrix[3]. Moreover the wavelength of the quantum well optical transition can be tuned by varying the indium composition to be above or below the photonic bandgap. In addition this design can be extrapolated to increase the lattice thickness, avoiding the complexity of scaling layers associated with a layer by layer process, traditionally used in 3D photonic crystals. A schematic of the designed growth structure is shown in Figure 1. 


\begin{tabular}{c} 
GaAs $225 \mathrm{~nm}$ \\
\hline AlGaAs \\
\hline GaAs $225 \mathrm{~nm}$ \\
\hline AlGaAs \\
\hline GaAs $225 \mathrm{~nm}$ \\
\hline AlGaAs \\
\hline GaAs $225 \mathrm{~nm}$ \\
\hline AlGaAs 225 \\
\hline nm \\
\hline GaAs Buffer
\end{tabular}

Figure 1: Schematic of the epitaxially grown structure for 3D photonic crystal. The structure consists of alternating layers of GaAs/AlGaAs which will form the lattice components of the photonic crystal in the growth direction.

\subsection{Semiconductor Process Flow Design}

A semiconductor processing flow, with minimal processing steps, reliability and scalability was designed and is under development. We envisage a process that includes multistep plasma etch, followed by oxidation of AlGaAs to realize the 3D photonic crystal. The key advantage of this process is the ability to generate multiple layers of the photonic crystal by a single step. The minimum feature size of the structure is $225 \mathrm{~nm}$ with aspect ratio of $8: 1$.

Once the material is grown, we perform a two dimensional (2D) nanopatterning with square lattice symmetry on the sample. Subsequently the pattern is transferred to GaAs-AlGaAs layer through a dry etch process. This process results in a square $2 \mathrm{D}$ pattern with deep etch to GaAs surface. This serves as a pathway to perform selective oxidation and subsequent etching later. Post etching the structure is then subjected to oxidation of the high aluminum content AlGaAs layers at an elevated temperature. The oxidation of GaAs is negligible in this process. This allows us to selectively convert the AlGaAs to AlOx. The presence of this intermittent GaAsAlOx structure provides us with the index contrast necessary for the photonic crystal structure. Once the GaAs-AlOx structure is formed, it is sculptured selectively through a wet etch process to have the desired filling factor. 


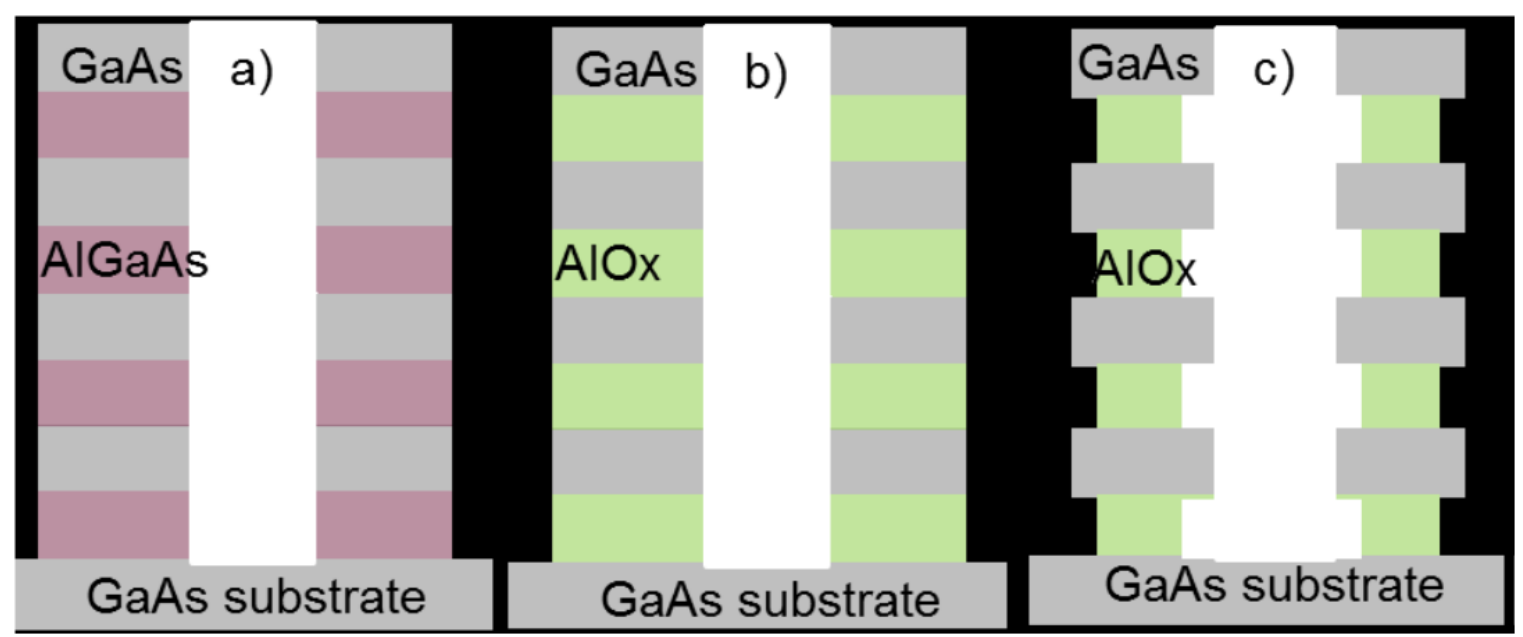

Figure 2. Process flow for the GaAs-AIGaAs photonic crystal: a) Denotes the dry etching process used to etch GaAs-AIGaAs epitaxially grown material b) Selective oxidation of AlGaAs layers to AlOx, c) Selective etch of AlOx layers.

\subsection{Semiconductor Processing}

Lithography: A high aspect ratio for etch necessitates the use of a hard mask for transferring electron-beam lithography patterns to optically active materials. Silicon dioxide was used as a hard mask onto which the lithographic pattern would be transferred and then subsequently to the GaAs-AlGaAs epitaxial layers. Since the features are sub-300 nm, and the wafers sizes are small, electron beam lithography was the chosen approach. The patterning was performed on a JEOL 9500FSZ, using Zeonrex (Corp.) Electron-beam Photoresist (ZEP) as the e-beam resist. ZEP provides good selectivity with respect to etching and the combination of ZEP+ underlying oxide layer provides a good etch mask for deep etching GaAs- AlGaAs. An image of the e-beam lithographic process is shown in Fig. 3.

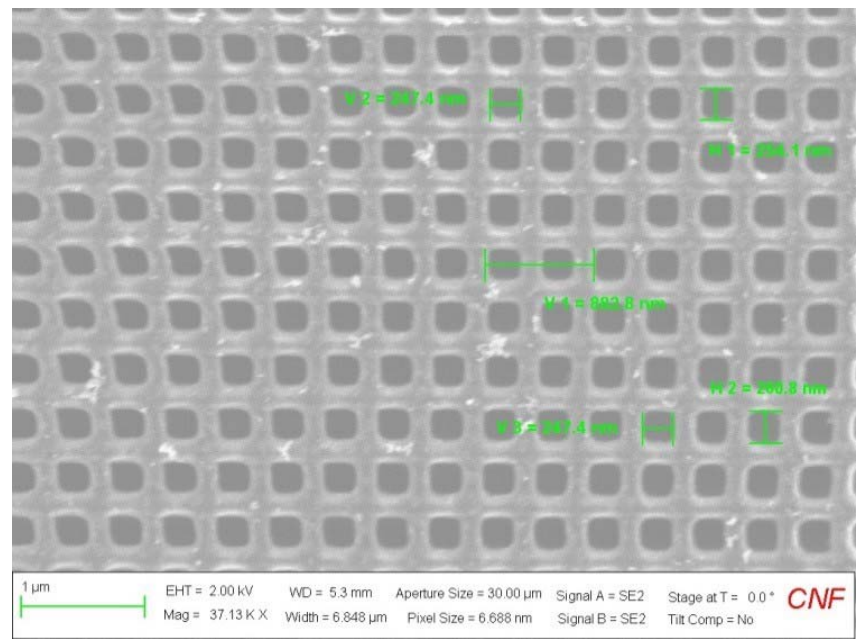

Figure 3: Scanning electron microscopy (SEM) image of the fabricated 2D structure using electron beam lithography. ZEP was used as the electron beam resist. 
Etching: In order to successfully transfer patterns from the resist to the oxide and from the oxide to GaAs - AlGaAs, etching techniques for selectively removing $\mathrm{SiO}_{2}$ over $\mathrm{GaAs}$, and GaAs over $\mathrm{SiO}_{2}$ were developed as part of this project. The recipes developed are highly robust with selectivities of over 20:1, and provide a valuable tool for future III-V based developments.

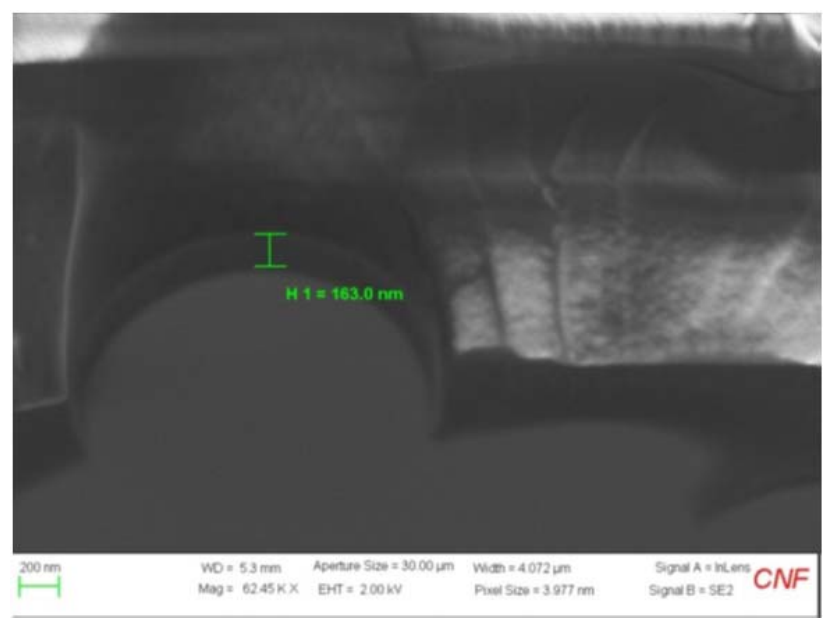

Figure 4. SEM image of structure after $\mathrm{SiO}_{2}$ etch process calibration. The residual photoresist, $\mathrm{SiO}_{2}$, and underlying $\mathrm{GaAs}$ layer can be seen. A $\mathrm{CHF} 3 / \mathrm{O}_{2}$ based etch process was used.

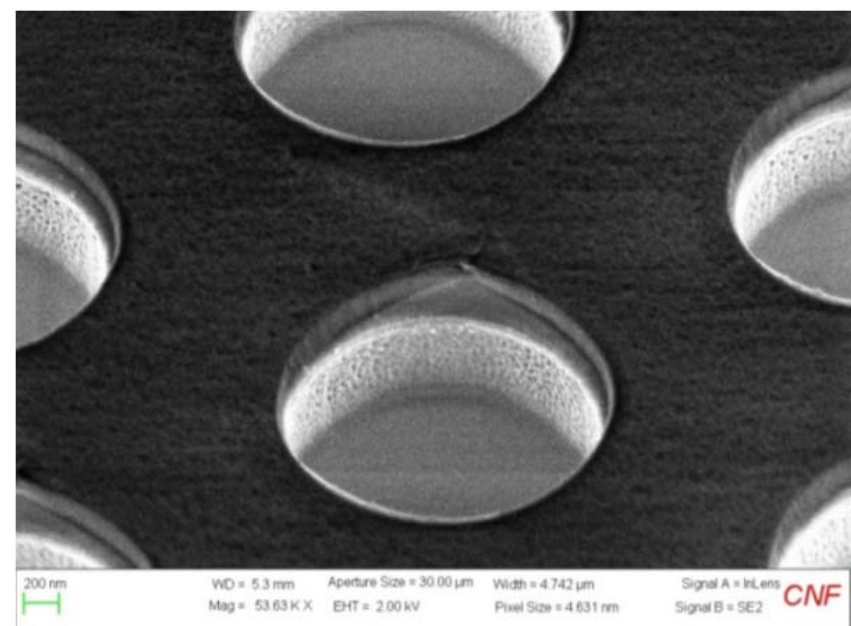

Figure 5. Image of the sample after GaAs etch calibration. An intact hard mask $\left(\mathrm{SiO}_{2}\right)$ and the etched GaAs layer is seen

In order to be cost-conscious all etch process developments were made on photolithography patterned GaAs wafers, instead of using electron beam lithography. A CHF3/O $\mathrm{O}_{2}$ etch process was developed for etching $\mathrm{SiO}_{2}$ which is highly selective with respect to the GaAs layer. It complements the $\mathrm{BCl}$ /Ar chemistry-based process used for etching GaAs which has a high degree of etch resistance for $\mathrm{SiO}_{2}$. A combination of these processes ensures a highly reliable overall pattern transfer. 


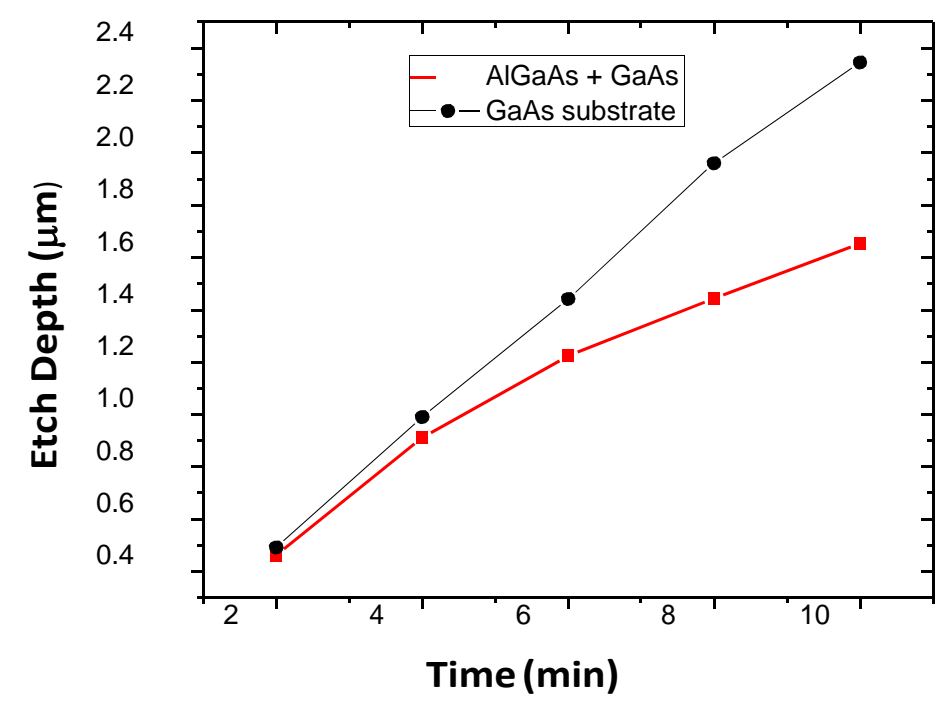

Figure 6: Etch rate variation of GaAs calibration samples and the epitaxially-grown GaAsAlGaAs samples under dry etch process.

It was observed that the etch rate of GaAs-AlGaAs samples are lower than GaAs substrate used for calibration. This will be accounted for while etching actual e-beam lithography sample or higher Ar concentration would be used to increase milling.

Oxidation: The presence of high aluminum content in the AlGaAs layers makes it selectively possible to oxidize $\mathrm{AlGaAs}$ into $\mathrm{AlO}_{\mathrm{x}}$. The presence of $\mathrm{GaAs}-\mathrm{AlO}_{\mathrm{x}}$ intermittent structure provides this design with the index contrast necessary to produce a photonic band gap in this system. A steam-based oxidation system has been found to produce $\mathrm{AlO}_{\mathrm{x}}$ with sufficient mechanical strength, lower stress and optical properties necessary for optical use [4].

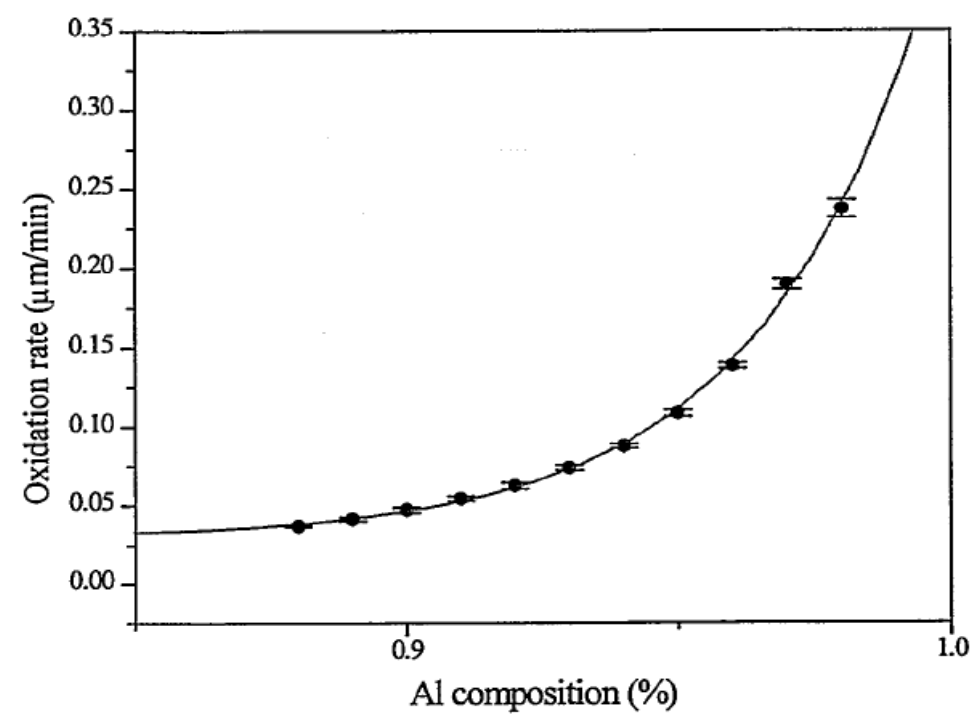

Figure 7: Oxidation rate of AlGaAs as function of composition. The temperature is kept constant at $400 \mathrm{C}[4]$. 
Different oxidation conditions including variation of carrier gases and temperatures were carried out. The presence of steam as an oxidation agent was observed to be vital as opposed to little or no oxidation in the presence of Oxygen alone. The oxidation was carried out on a furnace at a temperature of $420 \mathrm{C}$, for 20 minutes. The steam oxidizing agent was passed through the chamber using $\mathrm{N}_{2}$ as carrier gas. The steam bubble temperature and the flow rate were kept constant.

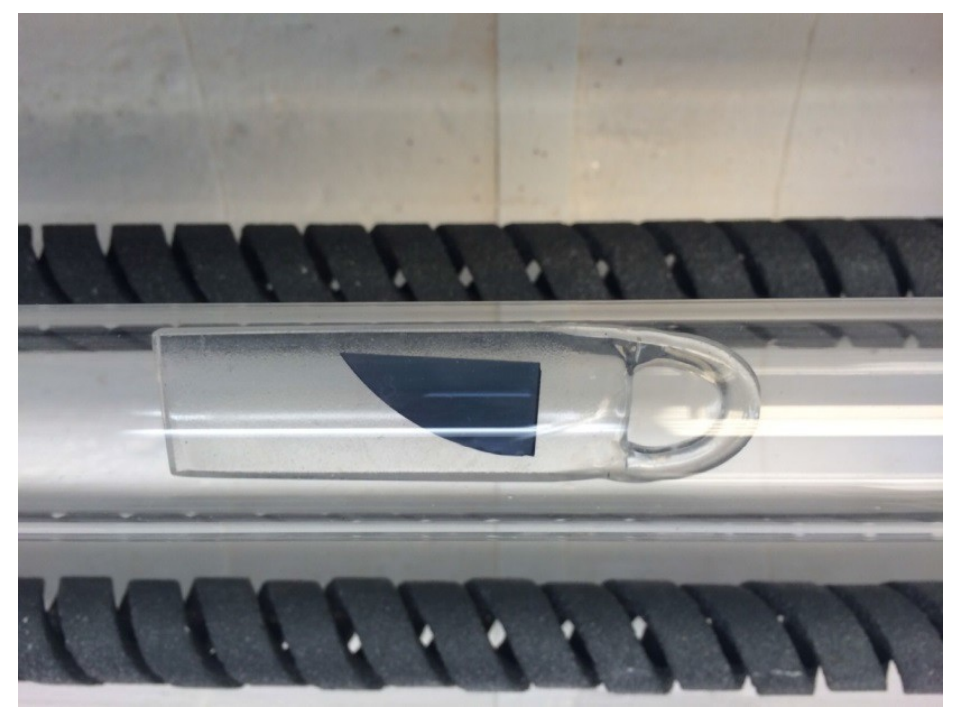

Figure 8: Steam oxidation of AlGaAs into GaAs. The samples are kept inside a quartz boat and $\mathrm{N} 2$ is used as a carrier gas.

The sequence of semiconductor fabrication was successfully executed and photonic crystal samples were fabricated with GaAs-AlOx as constituents. An illustrative SEM image of the structure formed is shown in Fig. 8. It is observed that the edges of the lattice are rough. This is primarily from the $\mathrm{ZEP}-\mathrm{SiO}_{2}$ pattern transfer process. This process would be optimized through improving the e-beam lithography and use of a hard mask alone while transferring patterns to GaAs. 


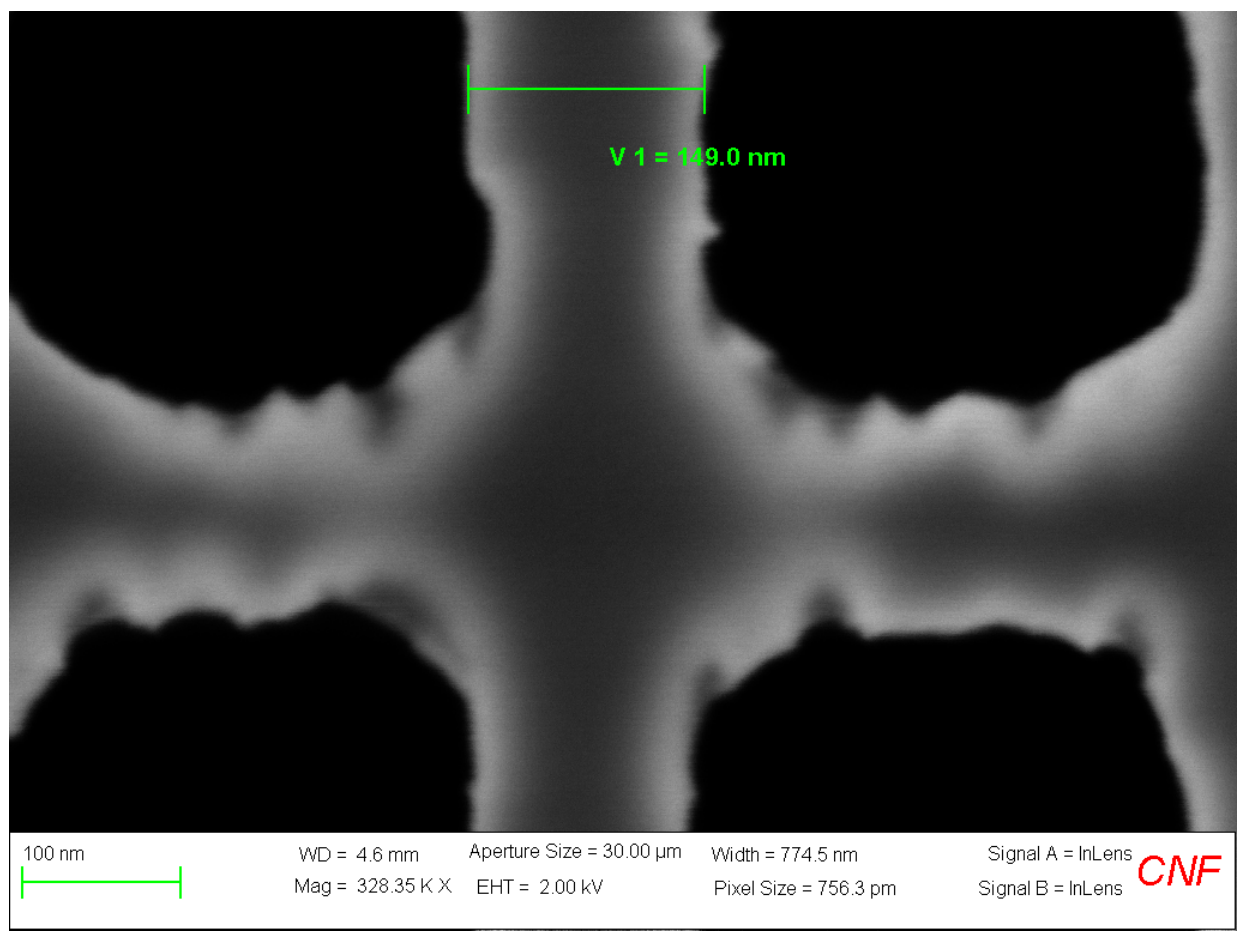

Figure 9: SEM image of a lattice of the initial GaAs-AIGaAs photonic crystal sample fabricated.

\subsection{CONCLUSIONS AND FUTURE WORK}

This project has provided innovative pathways to design 3D photonic crystals through processes with minimal complexity and fabrication steps. The use of III-V materials, with potential to embed optically active quantum well/ quantum dot structures, provides a tool to study fundamental phenomena related to light-matter coupling with in completely confined systems. Reliable design and semiconductor fabrication techniques were developed for 3D photonic crystals using the GaAs-AlGaAs material system. A set of selective dry etch processes with high selectivity were developed to etch $\mathrm{GaAs}-\mathrm{AlGaAs}$ with $\mathrm{SiO}_{2}$ as hard mask. Optical characterization of samples fabricated using variable angle transmission and reflection would be performed moving forward to ascertain the nature of the band gap. An optimal wet etch would be developed to sculpture the fabricated structure to obtain the photonic band gap. These two processes would be cycled through to nail a reproducible process for measuring a photonic bandgap. 


\section{REFERENCES}

1. R. Biswas, et al., "Three dimensional photonic band gaps in modified simple cubic lattices," Physical Review B, 65, 205121 (2002).

2. E. Chow, et al., "Three dimensional control of light in a 2D photonic crystal slab," Nature 407, pp. 983-986 (2000).

3. O. B. Spahn, et al., "Selective Oxidation Technology and its Applications toward Electronic and Optoelectronic Devices," SAND99-0921, July 1999.

4. R. L. S. Devine, "Photoluminescence characterization of InGaAs/GaAs quantum well Structures," Semicon. Sci. Tech, 3 (1988). 


\section{ACRONYMS}

$2 \mathrm{D}$

$3 \mathrm{D}$

JEOL

MBE

SEM

ZEP
Two Dimensional

Third Dimensional

Japan Electron Optics Laboratory

Molecular beam epitaxy

Scanning Electron Microscopy

Zeonrex (Corp.) Electron-beam Photoresist 


\section{DISTRIBUTION LIST}

DTIC/OCP

8725 John J. Kingman Rd, Suite 0944

Ft Belvoir, VA 22060-6218

$1 \mathrm{cy}$

AFRL/RVIL

Kirtland AFB, NM 87117-5776 2 cys

Official Record Copy

AFRL/RVSS/David Cardimona 1 cy 
(This page intentionally left blank) 